\title{
New method for evaluation of in vivo scattering and refractive index properties obtained with optical coherence tomography
}

\author{
A. Knüttel \\ ISIS Optronics GmbH \\ Innstr. 34 \\ 68199 Mannheim, Germany \\ E-mail: a.knuettel@isis-optronics.de
}

\section{S. Bonev}

W. Knaak

Fachhochschule Mannheim

Windeckstr. 110

68163 Mannheim, Germany

\begin{abstract}
Optical coherence tomography (OCT) provides more parameters than pure morphology does. In a recent paper [A. Knuettel and M. Boehlau-Godau, J. Biomed. Opt. 5(1) 83-92 (2000)] we have shown that the refractive index (RI) can be evaluated in a localized manner in skin tissue under in vivo conditions. Based on a theory, originally developed for light detecting and ranging applications [L. Thrane et al., J. Opt. Soc. Am. A 17(3) 484-490 (2000)], the parameter mean scattering angle (MSA) could be derived in addition to RI. The effects of hydration on MSA and RI have been evaluated in vitro in pigskin and in vivo in human skin with our OCT scanner SkinDex $300 \circledast$. These parameters may have a viable impact in (cosmetic) skin research and clinical diagnoses. To the best of our knowledge, this is the first time that (multiple) scattering of light has been quantified through the observation of a new scattering parameter under in vivo conditions. ๑ 2004 Society of Photo-Optical Instrumentation Engineers.

[DOI: $10.1117 / 1.1647544]$
\end{abstract}

Keywords: OCT; SkinDex $300^{\circledR}$; refractive index; mean scattering angle; in vivo; skin.

Paper 014002 received Feb. 10, 2003; revised manuscript received Jun. 3, 2003; accepted for publication Jun. 5, 2003.

\section{Introduction}

Optical coherence tomography (OCT) has become important in noninvasive medical diagnostics. Since an early paper was published more than 10 years ago, ${ }^{1}$ high resolution images of morphological microstructures in biological systems have been continuously improved especially in quality. Applications range from retinal macular diseases ${ }^{2}$ and optically moderately scattering tissue of the body's interior such as the gastrointestinal tract ${ }^{3}$ to highly scattering tissue like skin. ${ }^{4,5}$

OCT works analogously to an ultrasound scanner; the major difference is that ultrasound pulses are replaced by broadband light. The corresponding short coherence length permits a spatial resolution in depth direction of less than $10 \mu \mathrm{m} .{ }^{1}$ The lateral resolution is given by the focusing power of the employed objective as long as single scattering prevails. Depending on the scattering properties of tissue and some accepted loss in resolution, a penetration depth of about $1 \mathrm{~mm}$ can be achieved.

OCT is capable of probing other interesting parameters besides morphology in tissue. Water absorption has been evaluated by tuning the light source to a harmonic band at $1.46 \mu \mathrm{m} .{ }^{6}$ Birefringence for the characterization of ordered structures from collagen and elastin fibers has been exploited. ${ }^{7}$ Doppler flow imaging in vessels becomes increasingly popular because of the inherent sensitivity to frequency shifts in the detected OCT signals. ${ }^{8}$ In addition, we recently demonstrated the depth-dependent evaluation of the parameter refractive index (RI) in human skin. ${ }^{9}$ The RI is measured as

Address all correspondence to A. Knüttel, Tel: +49 (0)621-84251-0; Fax: +49 (0)621-84251-20; E-mail: a.knuettel@isis-optronics.de geometrical mean of the phase and group refractive indices.

The important parameters for differentiation and light propagation in tissue are RI and scattering. Both parameters are related to each other in a complicated manner. Scattering is the end result of local RI variations. Depending on the local geometry, certain phase functions arise, which can be reduced to a mean scattering angle (MSA) for mathematical simplification. In simple cases of spherical geometries, homogeneous RI inside and a different RI outside a sphere, the analytical solutions for the phase function and MSA are available from Mie theory. ${ }^{10}$ When the single-scattering regime with purely ballistic photons is not valid anymore, the detected signal loss versus depth is not just dependent on the uncorrected scattering coefficient $\mu_{s}$ but it becomes a complicated function of MSA and other physical and technical parameters. ${ }^{11}$ Particularly, small MSAs cause less detected signal loss due to the contributions from significant multiple forward scattering.

A certain area of interest within the probe has to be averaged to obtain meaningful data in MSA and RI. There are two reasons for this: (a) Tissue is too inhomogeneous for accurate spot measurements on cell size level, and (b) interference between backreflected signals from various points at mutual distances on the order of the coherence length prevents stable signals. Particularly the partially or completely destructive signals from issue (b), which are called speckles, generate significant variance in the detected signal. Incoherent averaging of signal intensities over a certain spatial region within the computer is very effective in reducing this variance.

1083-3668/2004/\$15.00 ㄷ 2004 SPIE 


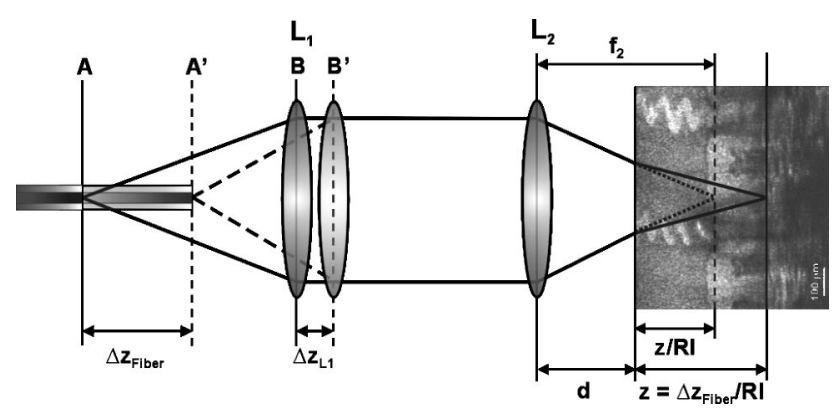

Fig. 1 OCT setup

The scope of this paper is to perform quantitative and simultaneous measurements of the parameters MSA and RI of skin under in vitro and in vivo conditions. The paper is organized as follows: In Sec. 2, the OCT setup is outlined and the crucial technical parameters are presented. In Sec. 3, the basic formulas of the underlying theory ${ }^{11}$ are recapitulated and adapted for the evaluation of our data. Experiments in turbid media are presented to reveal deviations from ideal ballistic light propagation conditions due to multiple forward light scattering. Based on those data, the parameters MSA and RI are evaluated and compared with theory in Sec. 4. In Sec. 5, the experimental data from pig and human skin are presented and discussed. A summary and conclusion follows in Sec. 6.

\section{OCT Setup}

In this paper, only the crucial part of the optical setup from the OCT device for simultaneous measurements of MSA and RI is described. A typical setup of a complete OCT scanner is presented and explained elsewhere. ${ }^{1}$ The core of the imaging setup consists of a Michelson interferometer, employing four optical arms. In Fig. 1 the main hardware features for the parameter measurements are shown in the sample arm. The optical path in the reference arm (not shown) remains constant, while only the distal tip of a fiber in the sample arm keeps on moving. The setup and functionality of RI measurements have already been described in our recent paper. ${ }^{9}$ Thus only the major issues are briefly recapitulated here.

To obtain RI data, the axial focus condition, which defines the amplitude of the backreflected signal in a turbid medium and the interference condition, which defines the actual position of the depth signal, have to be considered. A maximum backreflected and ensemble averaged signal is acquired by shifting the axial focus to the interference position. The shift is dependent on the (spatially varying) RI within the probe. We refer to this condition as the focus tracking mode. Under the assumption of constant RI for a moment, the ratio of the focal lengths of both lenses in the sample arm can be chosen to match the RI. In this case, moving the distal tip of the fiber will move the focus exactly parallel to the interference position, while the position of lens 1 can be kept stationary. Please notice that lens 2 always stays in a fixed position. Any local deviation from the mean RI requires a comparably small shift in the position of lens 1 to ensure focus tracking. Short confocal zones of lens 1 and 2, due to high numerical apertures (NA), facilitate the focus tracking procedure. Choosing the lens 1 position at maximum signal versus depth information provides the depth dependent RI data.

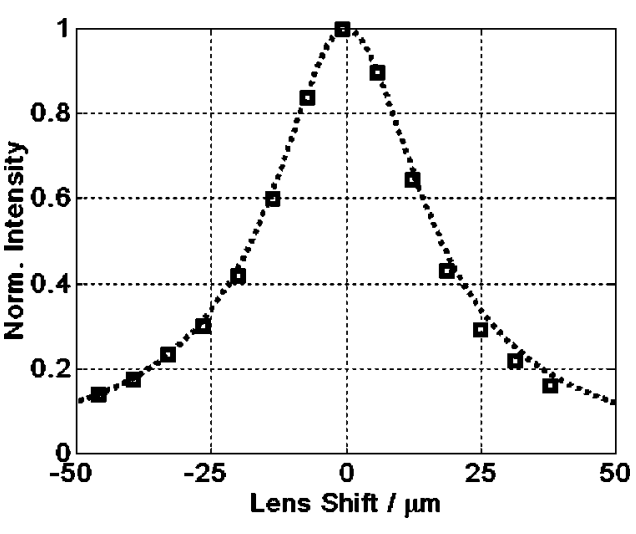

Fig. 2 Normalized squared heterodyne signal current (suspension).

To obtain the MSA, the same setup and technical parameters were employed. In contrast to the RI evaluation, ensemble averaged signal intensities at all lens 1 positions have to be acquired. The two-dimensional (2-D) map of the signals as function of lens 1 position and depth provides the MSA data.

The performance data of our OCT system SkinDex $300^{\circledR}$ with regard to technical parameters are as follows: A bandwidth of $\Delta \lambda=100 \mathrm{~nm}$ and a center wavelength of $\lambda_{0}$ $=1300 \mathrm{~nm}$ generated a coherence length of about $5.3 \mu \mathrm{m}$ in tissue. An average RI of either 1.41 or 1.42 for a sample medium of suspensions or tissue, respectively, has been assumed and corresponding objectives for the scanner have been selected. To ensure optimal focus tracking, the ratio of the focal lengths within the objectives was set to define a corresponding device parameter to be $n_{\mathrm{obj}}=f_{1} / f_{2}=1.41$ or 1.42. For a two-dimensional image, the field of view covers about $0.9 \mathrm{~mm}$ in depth (500 pixels) and $1 \mathrm{~mm}$ in one lateral direction (512 pixels). Several two-dimensional data sets (six in total) along the second lateral direction at an interplane distance of $10 \mu \mathrm{m}$ were acquired to compute an averaged 2-D data set. In addition, 14 different lens 1 positions have been selected to provide the focus dimension. These multidimensional data sets, which are comprising the averaged 2-D data at each lens 1 position, are to be obtained within 3-4 min. This acquisition time is orders of magnitudes larger than the correlation times of Brownian motion which is on the order of $10 \mathrm{~ms}$ for $\mu \mathrm{m}$ size particles. ${ }^{12}$ Thus it can be assumed that temporal microstructural changes in RI average out to a mean value.

The length of the confocal zone from the focusing power of the objective was extracted from Fig. 2, where the ensemble averaged, backscattered signal close to the surface of a turbid homogeneous medium was plotted. Given the definition of full width at half maximum (FWHM), a confocal zone of $\mathrm{FWHM}=35 \mu \mathrm{m}$ was extracted. Mathematical simplicity indicates that the confocal zone needs to be large compared to the coherence length of $5.3 \mu \mathrm{m}$. The spatial resolution in both lateral directions was measured to be about $3 \mu \mathrm{m}$.

\section{Theory}

For the subsequent description of the equations regarding MSA and RI, polarization and absorption effects have been neglected. In a recent paper $^{9}$ multiple scattering arising at 
penetration depths exceeding a few hundred $\mu \mathrm{m}$ was ignored for simplicity, while here it is the crucial issue for the MSA evaluation. It requires an entire two-dimensional data set (signal intensity as function of lens 1 position and depth) while for the RI evaluation a subset or one-dimensional plot (lens 1 position at maximum signal intensity versus depth) is sufficient. In the following sections, evaluations for MSA and RI are given.

\subsection{MSA Evaluation}

The underlying theory has already been described in the literature. ${ }^{11}$ Based on the mutual coherence function of a multiply scattered object beam and an undisturbed reference beam, the following mean square signal heterodyne current as function of the depth $z$ was obtained:

$$
\begin{aligned}
\left\langle i^{2}(z)\right\rangle= & \frac{K}{\pi w_{H}^{2}(z)}\left[e^{-2 \mu_{S} z}+\frac{2 e^{-\mu_{S} z}\left(1-e^{-\mu_{S} z}\right)}{1+\frac{w_{S}^{2}(z)}{w_{H}^{2}(z)}}\right. \\
& \left.+\left(1-e^{-\mu_{S} z}\right)^{2} \cdot \frac{w_{H}^{2}(z)}{w_{S}^{2}(z)}\right] \\
\equiv & \left\langle i^{2}(z)\right\rangle_{0} \Psi(z) .
\end{aligned}
$$

The terms in the large bracket define the heterodyne efficiency factor $\Psi(z)$, which describes the signal loss due to single and multiple scattering. The mean scattering coefficient $\mu_{S}$ within the turbid medium is responsible for the signal decay independent of the beam geometry and multiple scattering. The constant $K$ comprises the signal powers in the sample and reference arm as well as the backscattering coefficient in the absence of scattering.

Important parameters in Eq. (1) are the radii $w_{H, S}(z)$ where the intensity signal drops to $1 / e$ of the initial value in the center of the beam. The radius $w_{H}$ covers the unperturbed beam, while $w_{S}$ is influenced by multiple scattering as given below

$$
\begin{gathered}
w_{H}^{2}(z)=w_{0}^{2}\left(1-\frac{B(z)}{f_{2}}\right)^{2}+\left(\frac{B(z)}{k w_{0}}\right)^{2} \text { and } \\
w_{S}^{2}(z)=w_{H}^{2}(z)+\left(\frac{2 B(z)}{k \rho_{0}(z)}\right)^{2} .
\end{gathered}
$$

In Eqs. (2), $w_{0}$ is defined as the radius where the intensity of the unperturbed beam in the plane of lens 2 drops to 1/e compared to the value on the optical axis. Without loss of generality regarding the spectral width employed, only the center wavelength $\lambda_{0}$ of the quasimonochromatic spectrum needs to be considered, which defines the propagation constant to $k=2 \pi / \lambda_{0}$ The ray matrix element $B(z)=d+z / \mathrm{RI}, d$ being the free space distance from lens 2 to the sample surface, and the focus length $f$ are given relative to air in the original paper. $^{11}$

The variables $z$ and $f$ have to be related to the two experimental variables $\Delta z_{\text {Fiber }}$ and $\Delta z_{L 1}$ of our setup shown in Fig. 1. According to our recent paper, ${ }^{9}$ the interference condition defines the probed depth at

$$
z=\frac{\Delta z_{\text {Fiber }}}{\text { RI }}
$$

This leads to the ray matrix element

$$
B(z)=d+\frac{\Delta z_{\text {Fiber }}}{\mathrm{RI}^{2}}
$$

while the focus length of lens 2 in air is given by

$$
f_{2}=d+\frac{\left(\Delta z_{\text {Fiber }}-\Delta z_{L 1}\right)}{n_{\mathrm{obj}}^{2}} .
$$

Unlike the original paper, ${ }^{11}$ here the effective focal length varies slightly to accommodate the focus shift according to the probed depth (interference condition). Under the influence of multiple forward scattering which is described by a finite coherence length $\rho_{0}(z)$ (described below), the beam waist in Eq. (2b) increases as compared to Eq. (2a). In the case of an infinite coherence length $\rho_{0}$ or a perfectly mutually coherent beam, Eqs. (2a) and (2b) reduce to the same form $w_{H}(z)$ $=w_{S}(z)$.

The ratio $w_{0} / f_{2}$ or the numerical aperture NA are needed to evaluate Eqs. (2). According to the equations given above, the signal intensity in the absence of scattering (e.g., close to the surface of a sample) can be approximated by

$$
\left\langle i^{2}(z=0)\right\rangle \propto \frac{1}{w_{H}^{2}(z=0)} \propto \frac{1}{1+\left[\frac{\Delta z_{L 1}}{n_{\mathrm{obj}}^{2}} \cdot \frac{k w_{0}^{2}}{f_{2}^{2}}\right]^{2}} .
$$

Figure 2 shows the experimental data and a fit of a theoretical curve according to Eq. (6). To ensure strong backscattering, a suspension with $2.1 \mu \mathrm{m}$ polystyrene particles was measured directly below the surface. At a slice thickness of only $20 \mu \mathrm{m}$, multiple forward scattering could be ignored even at a high scattering coefficient of $17 \mathrm{~mm}^{-1}$. Otherwise the same experimental parameters were used as described in Sec. 2. According to Eq. (6), the FWHM $=35 \mu \mathrm{m}$ results in $w_{0} / f_{2}=0.154$.

For Gaussian optics, the following relation between the $\mathrm{NA}$, the ratio $w_{0} / f_{2}$ and the spot radius $w_{H \text {, min }}$ in the focal plane $\left(B=f_{2}\right)$ is valid ${ }^{13}$

$$
w_{H, \min }=\frac{f_{2}}{k w_{0}}=\frac{\lambda_{0}}{\pi \cdot \mathrm{NA}} .
$$

Equation (7) yields a NA $=0.31$. The corresponding minimum spot diameter of $2 w_{H, \min } \approx 2.7 \mu \mathrm{m}$ is in close agreement with the measured value.

In addition, for the evaluation of Eq. (2b), the lateral coherence length and the MSA are related as follows ${ }^{11}$ :

$$
\rho_{0}(z)=\sqrt{\frac{3}{\mu_{S} z}} \cdot \frac{\lambda}{\pi \cdot \mathrm{MSA}} \cdot\left(1+\frac{\mathrm{RI} \cdot d_{\text {mix }}}{z}\right),
$$

where the RI and the scattering coefficient $\mu_{s}$ within the turbid medium need to be known. Strictly speaking, RI and $\mu_{s}$ are also functions of depth. For mathematical simplicity, however, we always assume averaged RI and $\mu_{s}$ values within the object. The last factor in Eq. (8) arises from the fact that the 


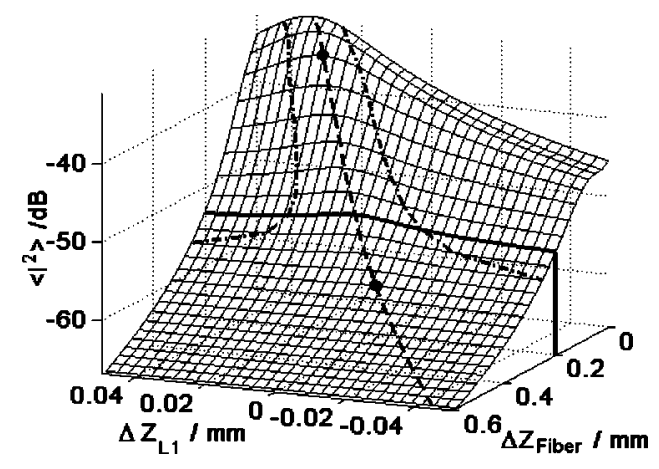

Fig. 3 Squared heterodyne current (theory).

lateral coherence length of a wave increases while traveling a distance $d_{\text {mix }}$ without being perturbed by scatterers. This is known as the so-called "shower curtain effect:" one can roughly see a person behind a shower curtain if one is not too close to the curtain itself. In our setup, this distance $d_{\text {mix }}$, the position of the heterodyne mixing plane, lies around the position of lens 2 and will be determined later experimentally. This theory does not account for speckle effects which are apparent in our data. However, it is possible to reduce these effects to an acceptable level by averaging along the second lateral dimension.

The square signal heterodyne current in Eq. (1) is a function of the measured variables $\Delta z_{\text {Fiber }}$ and $\Delta z_{L 1}$, the sample parameters RI, MSA and $\mu_{s}$ and the device constants $\lambda_{0}, f_{2}$, $w_{0}, n_{\text {obj }} d$ and $d_{\text {mix }}$. Figure 3 shows an example of this function corresponding to the material parameters $\mathrm{RI}=1.34$, $\mathrm{MSA}=5^{\circ}$ and $\mu_{s}=19 \mathrm{~mm}^{-1}$. The slope of the diagonal line, which is defined by the lens 1 positions of the maximum of $\left\langle i^{2}\right\rangle$ at given $\Delta z_{\text {Fiber }}$ values in the $\Delta z_{\text {Fiber }}-\Delta z_{L 1}$-plane, is only determined by the RI value. This RI is evaluated first (see below). The graph reveals that the signal loss at tracked focus condition is a nonlinear function (on a log scale) of depth or fiber position $\Delta z_{\text {Fiber }}$. Thus an effective scattering coefficient $\mu_{\text {eff }}$ can be introduced defined by the signal drop between two different depth locations. Only in the absence of multiple scattering $\mu_{\mathrm{eff}}$ and $\mu_{s}$ are identical. The two adjacent lines depict the lens 1 positions, at which the signal has dropped by $1 \mathrm{~dB}$ compared to the corresponding maximum. Each depth dependent distance between the $1 \mathrm{~dB}$ points defines a so-called "scatter width." Just beneath the surface, this width is only determined by the NA of the beam [see Eqs. (6) and (7)] but it increases at larger depths due to multiple forward scattering.

In the next figure (Fig. 4), it is shown how the experimental values, scatter width (sw) and effective scattering coefficient $\mu_{\text {eff }}$ depend on both of the sample parameters, $\mu_{s}$ and MSA. Those relations are in turn a function of depth, so an arbitrary value of $\Delta z_{\text {Fiber }}=0.2 \mathrm{~mm}$ was chosen. Given the measured values sw and $\mu_{\text {eff }}$, one can find the appropriate sample scattering parameters MSA and $\mu_{s}$ at the intersection of the two corresponding contour lines.

The following observations can be made from Fig. 4: At MSAs $>7^{\circ}$ and $\mu_{s}<15 \mathrm{~mm}^{-1}$, the scattering coefficients $\mu_{\text {eff }}$ and $\mu_{s}$ are (almost) identical. Multiple scattering arises when those ranges are exceeded, which manifest in $\mu_{\text {eff }}<\mu_{s}$ or visibly in a nonlinear signal decay at log scale. At MSAs $<2^{\circ}$ and $\mu_{s}>10 \mathrm{~mm}^{-1}$, it is difficult to find the crossing

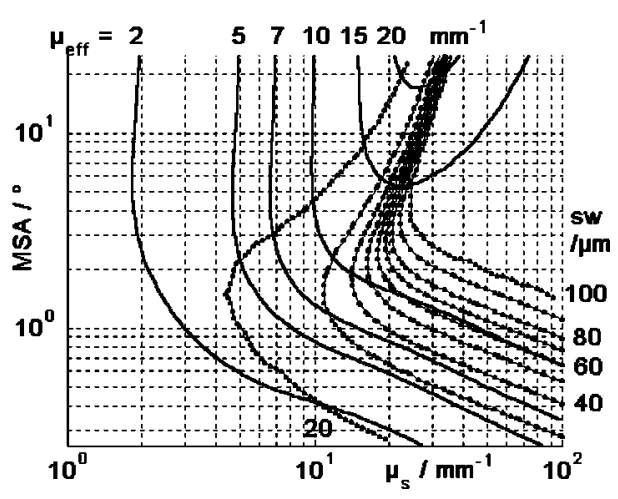

Fig. 4 Contour lines of the calculated scatter widths and effective scatter coefficients.

points since the contour lines for $\mu_{\text {eff }}$ and scatter width (sw) are aligned almost parallel, which generates larger error margins for the sample parameters.

\subsection{RI Evaluation}

The mean RI can be evaluated according to ${ }^{9}$

$$
\mathrm{RI}=\sqrt{n_{g} \cdot n_{p}}=\frac{n_{\mathrm{obj}}}{\sqrt{1-\left.\frac{\Delta z_{L 1}}{\Delta z_{\text {Fiber }}}\right|_{\text {focus }} ^{\text {fracking }}}} .
$$

The mean RI is the geometrical average of the group index $n_{g}$ and phase index $n_{p}$. The difference between both indices is usually a few percent and sufficiently small to be ignored for further treatment. For a piecewise homogeneous medium along depth direction, the slope $\Delta z_{L 1} / \Delta z_{\text {Fiber }}$ has to be evaluated at the focus tracked condition $\left(\Delta z_{L 1}\right.$ positioned for maximum signal). In this paper, however, only averaged values without depth dependency have been regarded.

Figure 5 shows the signal intensity normalized to its maximum value at each given depth $z$ versus lens 1 position for two different RIs. In case (a) a mixture of $75 \%$ water and $25 \%$ glycerin and in case (b) a $50 \%$ water and $50 \%$ glycerin mixture was used, yielding expected RIs of 1.38 and 1.41, respectively. For a given $2.1 \mu \mathrm{m}$ particle solution a scattering coefficient of about $6.2 \mathrm{~mm}^{-1}$ was measured. The fitted slopes of the maximum signals versus depth yielded the following experimental results: (a) $\mathrm{RI}=1.39$ and (b) $\mathrm{RI}=1.41$. This compares fairly well to the values computed from the mixtures. Please notice that in Fig. 5(b) no shift of lens 1 position was necessary to keep the detected signal at maximum for all depths. This special case of focus tracking is expected from Eq. (9) when RI matches the given device parameter of $n_{\mathrm{obj}}$ $=1.41$.

\section{Measurements with Polystyrene Suspensions}

To verify the validity of Eq. (1) for obtaining the MSA, $\mu_{s}$ and RI, respectively, various scattering suspensions with different scattering parameters have been measured. In Fig. 6, false color plots of the signal intensity normalized to its maximum value (per depth) versus focus position of lens 1 and depth are shown for scattering suspensions with two different 


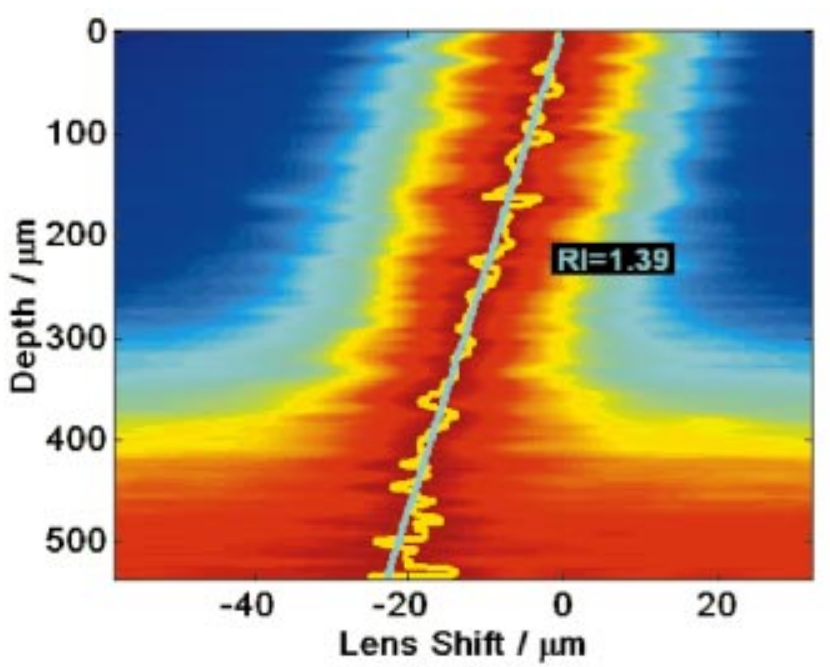

a)

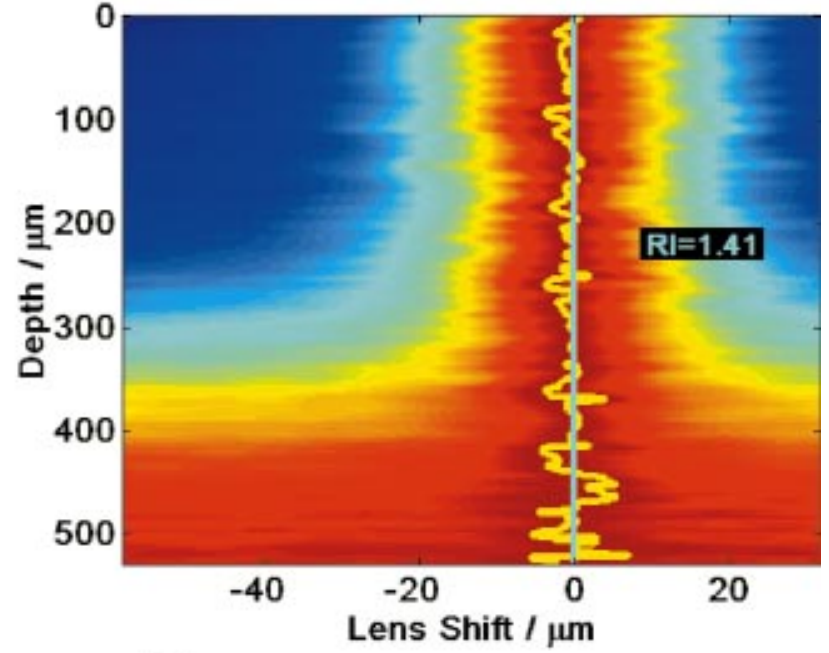

b)

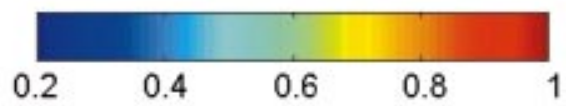

Fig. 5 False color plots of the normalized ensemble signal intensity (RI, suspension).

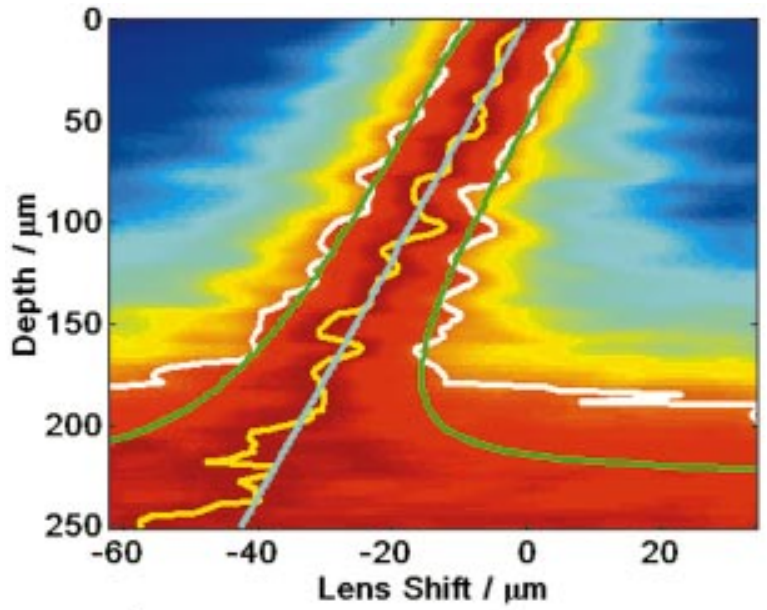

a)

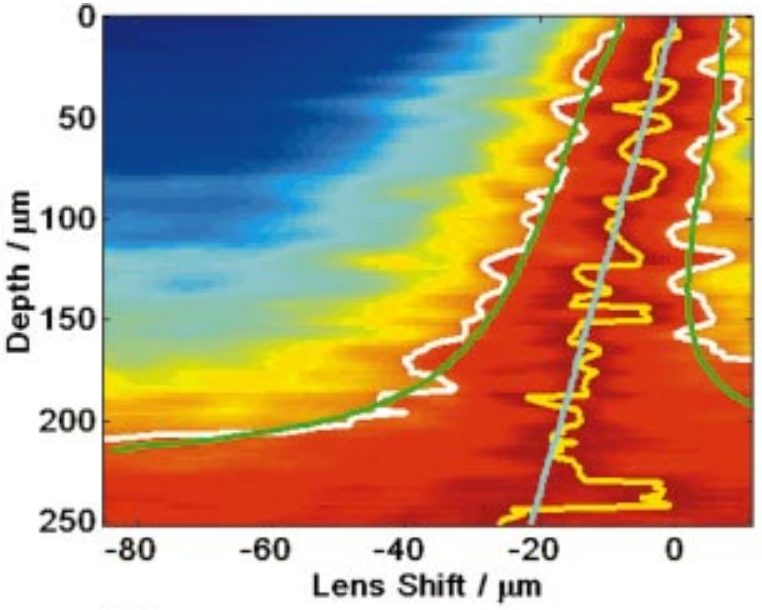

b)

Fig. 6 False color plots of the normalized ensemble intensity (MSA, suspension). 


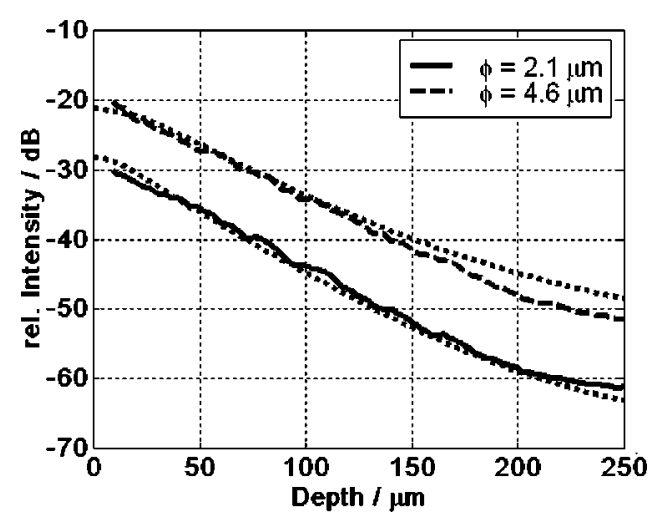

Fig. 7 Drop of the maximum intensity at focus tracking condition (suspension).

particle sizes [(a) $2.1 \mu \mathrm{m}$ and (b) $4.6 \mu \mathrm{m}$ ] in experiment and theory. Shown are the experimental (yellow) and fitted maximum intensity lines (blue) as well as the experimental (white) and fitted $-1 \mathrm{~dB}$ lines (green). Due to the electronic noise level at about $-60 \mathrm{~dB}$, the data around penetration depths of $200 \mu \mathrm{m}$ and beyond become incorrect. At those depths the strong apparent increase of the scatter widths is meaningless.

To obtain slightly smoothed functions, this and all subsequent data were interpolated by cubic spline functions. In addition, the RI values from the fitted portions of the corresponding data sets were employed for all figures to provide real (geometrical) depth scaling.

The maximum intensities as function of depth (focus tracking condition), i.e., the normalization factors from the data in Fig. 6, are shown in Fig. 7 for all samples together with the fitted curves according to theory. Expected and experimentally extracted parameters RI, MSA, $\mu_{\text {eff }}$ and $\mu_{s}$ are condensed in Table 1 . Within given uncertainties, the comparison shows a fairly good agreement between theory and experiment.

Larger particles with more pronounced forward scattering lead to a larger focus zone (linked to the scatter width) at increasing depths as seen in Fig. 6. Photons scattered from small size particles with large MSA are too incoherent to contribute to the detected interference signal.

The device constant "position of mixing plane $d_{\text {mix }}$ " is difficult to determine from technical data in an objective which has been shown in simplified form in Fig. 1. The following approach in extracting this device parameter, however, is a viable alternative. The goal is to match an extracted MSA to theoretical data from, e.g., Mie theory ${ }^{10}$ to provide $d_{\text {mix }}$. The MSA of the suspension with the $4.6 \mu \mathrm{m}$ spheres [see Fig. 6(b) ] yielded $d_{\text {mix }}=4.5 \mathrm{~mm}$, which was kept for all other evaluations. Given this value, the mixing plane and the lens 2 plane within the objective almost coincide.

\section{Skin Measurements}

Subsequently, data from in vitro pigskin and in vivo human skin are presented. In both cases three-dimensional data sets were acquired to provide sufficient signal averaging and speckle reduction within a region of interest. MSAs and RIs have been evaluated under different ambient conditions for the in vitro (Sec. 5.1) and in vivo (Sec. 5.2) studies. All except one of the technical parameters are as outlined above. By changing the objective, the device parameter slightly changed to $n_{\mathrm{obj}}=1.42$.

\subsection{Pigskin}

In Fig. 8, the normalized intensities versus focus positions and depths are shown for pigskin at two different ambient conditions. Under (b), the skin was treated for $20 \mathrm{~min}$ with a detergent solution which consists of $2 \%$ anionic tensides in water. As comparison, (a) shows untreated skin. In the treated case, the scatter width increases at much shallower depths as compared to the untreated situation. At a chosen depth of 200 $\mu \mathrm{m}$, a scatter width of about $90 \mu \mathrm{m}$ was found in the treated case as compared to $40 \mu \mathrm{m}$ in the untreated one. In addition, RI drops significantly due to the uptake of water.

The maximum intensities as function of depth (focus tracking condition) are shown in Fig. 9 for untreated and treated pigskin together with the fitted curves according to theory. The parameters are condensed in Table 1. The signal drop versus depth and the nonlinearity on log scale was more pronounced in the treated case. Analogous to the behavior of the scatter width, $\mu_{\mathrm{eff}} \approx 10.7 \mathrm{~mm}^{-1}$ in the treated case was higher than $\mu_{\text {eff }} \approx 8.1 \mathrm{~mm}^{-1}$ in the untreated one.

The data of the scatter widths and $\mu_{\text {eff }}$ 's yield quantitative MSA values for both ambient conditions according to the plot in Fig. 4. In the untreated case, we found a $\mathrm{MSA} \approx 1.5^{\circ}$ $\pm 0.5^{\circ}$ while in the treated case it only slightly rose to a

Table 1 Expected and experimentally extracted sample parameters.

\begin{tabular}{|c|c|c|c|c|c|c|c|}
\hline Sample & $\begin{array}{c}\text { Expected } \\
\text { RI }\end{array}$ & $\begin{array}{c}\text { Extracted } \\
\text { RI }\end{array}$ & $\begin{array}{l}\text { MSA }_{\text {Mie }} \\
{ }^{\circ} \text { (deg) }\end{array}$ & $\begin{array}{l}\mathrm{MSA}_{\text {extr }} \\
{ }^{\circ}(\mathrm{deg})\end{array}$ & $\begin{array}{l}\text { Expected } \mu_{s} \\
\mathrm{~mm}^{-1}\end{array}$ & $\begin{array}{c}\mu_{\text {eff }}, \text { extr } \\
\mathrm{mm}^{-1}\end{array}$ & $\begin{array}{c}\mu_{s}, \text { fitted } \\
\mathrm{mm}^{-1}\end{array}$ \\
\hline $\begin{array}{l}2.1 \mu \mathrm{m} \text { sphere } \\
\text { suspension }\end{array}$ & 1.37 & 1.345 & 12.4 & $\begin{array}{l}11 \\
\pm 2\end{array}$ & 18 & 19.4 & $\begin{array}{l}20 \\
\pm 1\end{array}$ \\
\hline $\begin{array}{l}4.6 \mu \mathrm{m} \text { sphere } \\
\text { suspension }\end{array}$ & 1.37 & 1.370 & 6.5 & $\begin{array}{r}5.5 \\
\pm 1.0\end{array}$ & 20 & 16.7 & $\begin{array}{l}17 \\
\pm 2\end{array}$ \\
\hline $\begin{array}{l}\text { Pigskin } \\
\text { (untreated) }\end{array}$ & $\cdots$ & 1.415 & $\cdots$ & $\begin{array}{c}1.5 \\
\pm 0.5\end{array}$ & $\cdots$ & 8.1 & $\begin{array}{r}10 \\
\pm 1\end{array}$ \\
\hline $\begin{array}{l}\text { Pigskin } \\
\text { (treated) }\end{array}$ & $\cdots$ & 1.365 & $\cdots$ & $\begin{array}{c}2.5 \\
\pm 0.5\end{array}$ & $\cdots$ & 10.7 & $\begin{array}{r}16 \\
\pm 2\end{array}$ \\
\hline
\end{tabular}




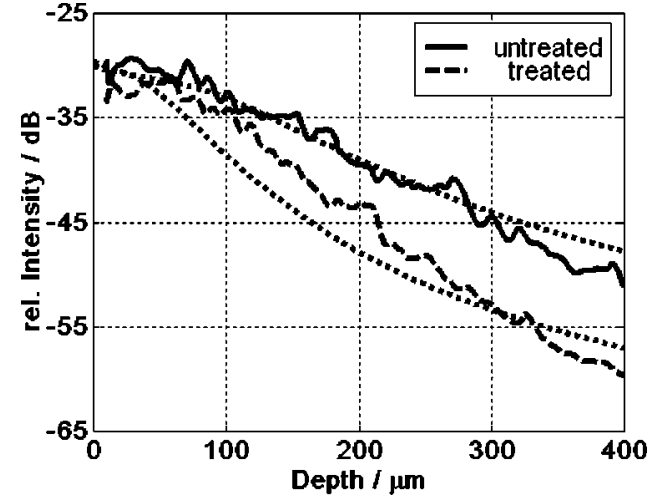

Fig. 9 Drop of the maximum intensity at focus tracking condition (pigskin).

$\mathrm{MSA} \approx 2.5^{\circ} \pm 0.5^{\circ}$, in spite of the strong increase of the scatter width. As shown in Fig. 4, the scatter width sw depends not only on the MSA but on the scattering coefficient $\mu_{s}$ as well. In this sample, the increase of the scatter width is in fact mostly due to the increase of the scattering coefficient $\mu_{s}$. The uptake of water, indicated by the strong drop of the RI, should not alter the average cell size to an extent that the MSA changes noticeably. A small increase of the MSA, however, is expected from Mie theory due to the change of the optical mismatch between the scattering particle and the surrounding medium.

\subsection{Human Skin}

In Fig. 10, the normalized intensities are shown for in vivo human skin at (a) untreated and (b) treated conditions. Similar to the previous experiment, skin on the volar site of the lower arm from a 26-year-old male volunteer was treated for $20 \mathrm{~min}$ with the same detergent solution (see Sec. 5.1). In contrast to pigskin in the treated case, the scatter width decreases rather than increases. The fact that the RI remains nearly constant around the epidermis $(20-120 \mu \mathrm{m})$ in both conditions reveals the physiological function of living as opposed to dead (pig) skin.

In Fig. 11(a), the data set from Fig. 10 has been processed to exhibit the corresponding scatter widths for both skin conditions. No fits to apparent MSAs were given, because within the investigated skin depth (up to $350 \mu \mathrm{m}$ ) human skin is much more heterogeneous than pigskin, as can directly be seen in OCT images. A fit which assumes a homogeneous turbid medium is questionable in human skin. Nevertheless, the data in both conditions are in line with an effective scattering coefficient in the untreated case of $\mu_{\mathrm{eff}} \approx 14 \mathrm{~mm}^{-1}$ and in the treated one of $\mu_{\mathrm{eff}} \approx 8 \mathrm{~mm}^{-1}$. Assuming the validity of Fig. 4, the corresponding MSAs for the untreated and treated cases can be retrieved to be $\mathrm{MSA} \approx 4^{\circ}$ and $2^{\circ}$, respectively. There seems to be a similar link between $\mu_{\text {eff }}$ and MSA as in the in vitro pigskin experiment. The reversed behavior from the in vivo versus in vitro studies, however, could not be explained.

\section{Summary and Conclusion}

It was demonstrated that our OCT scanner SkinDex 300® is able to provide additional physical parameters besides morphological imaging. The parameters' mean scattering angle (MSA) and refractive index (RI) have been measured according to the theoretical models ${ }^{11}$ in turbid homogeneous solutions and in vitro in pigskin as well as in vivo in human skin. Quantitative comparisons have been made for the turbid homogeneous polystyrene suspensions. To the best of our knowledge, this is the first time that (multiple) scattering of light has been quantified through the observation of a new parameter called scatter width under in vivo conditions.

The MSA for the homogeneous suspensions was evaluated with Mie theory. ${ }^{10}$ The fact that larger particles exhibit smaller MSAs than smaller ones could be verified clearly on an experimental basis. Also the absolute values in MSA agreed fairly well between theory and experiment. At increasing penetration depth, the larger size particle suspension exhibits a broader scatter width. This is expected because large particles with correspondingly small MSAs promote coherent forward propagation of nonballistic, multiply scattered photons to a larger extent.

In the multiple scattering regime, each of the experimentally accessible parameters' effective scattering coefficient $\left(\mu_{\text {eff }}\right)$ and scatter width (sw) depend on both sample parameters $\mu_{s}$ and MSA. It has been shown in Fig. 4 that for certain parameters, a larger $\mu_{\text {eff }}$ is always accompanied by an increase in scatter width while MSA is nearly unchanged. This has been shown in a quantitative and qualitative manner for the two skin measurements. Treating with detergent in the case of pigskin (in vitro) increased both parameters $\mu_{\text {eff }}$ and scatter width, while in the case of human skin (in vivo) the same treatment decreased both parameters. By treating (pig) skin with detergent we would not expect a significant change
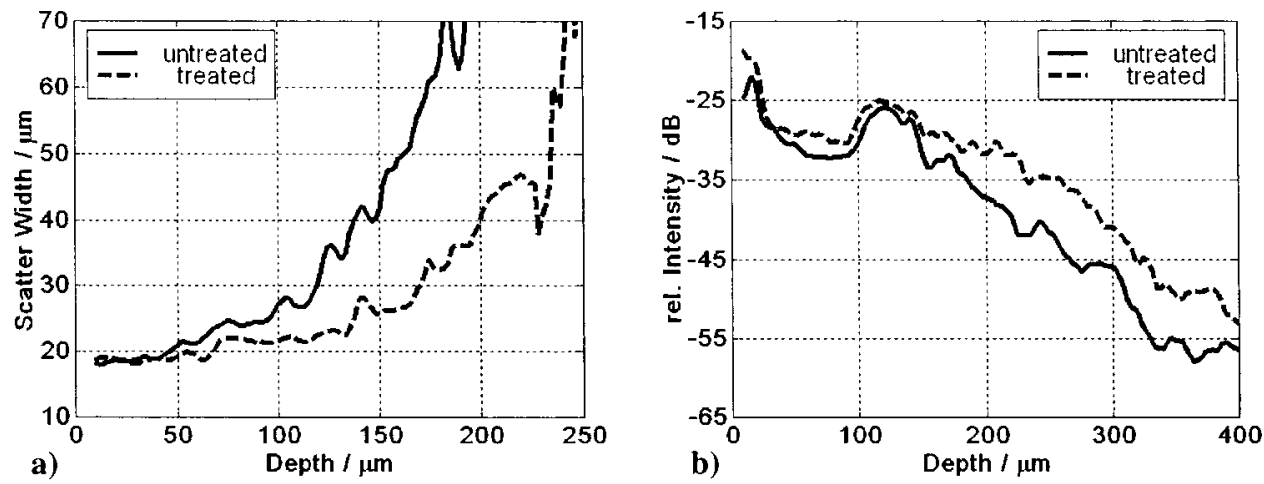

Fig. 11 Scatter width and signal decay (human lower arm). 


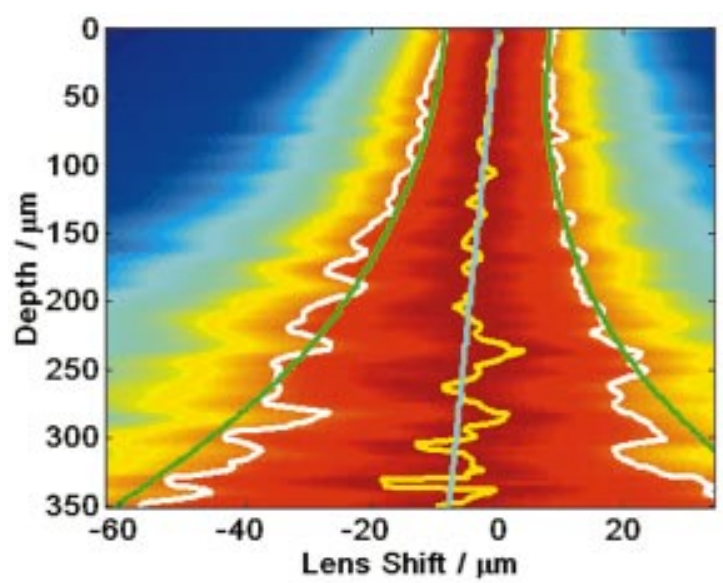

a)

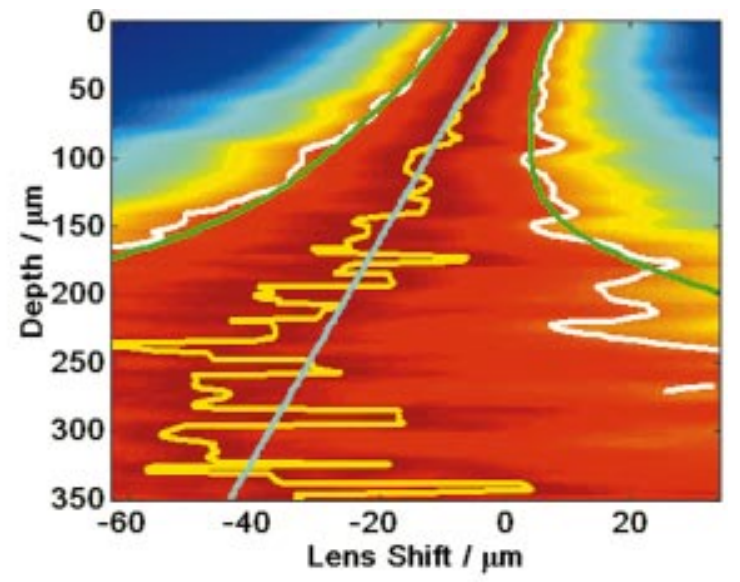

b)

Fig. 8 False color plots of the normalized ensemble intensity (pigskin).

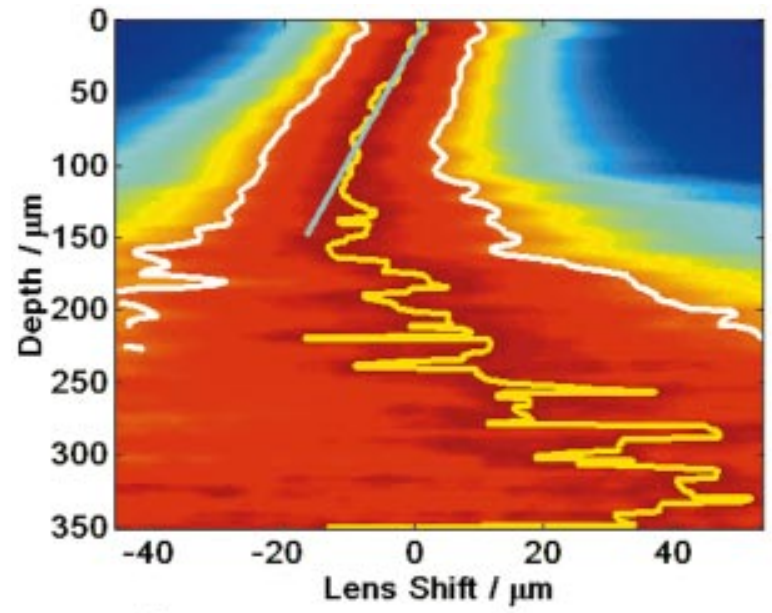

a)

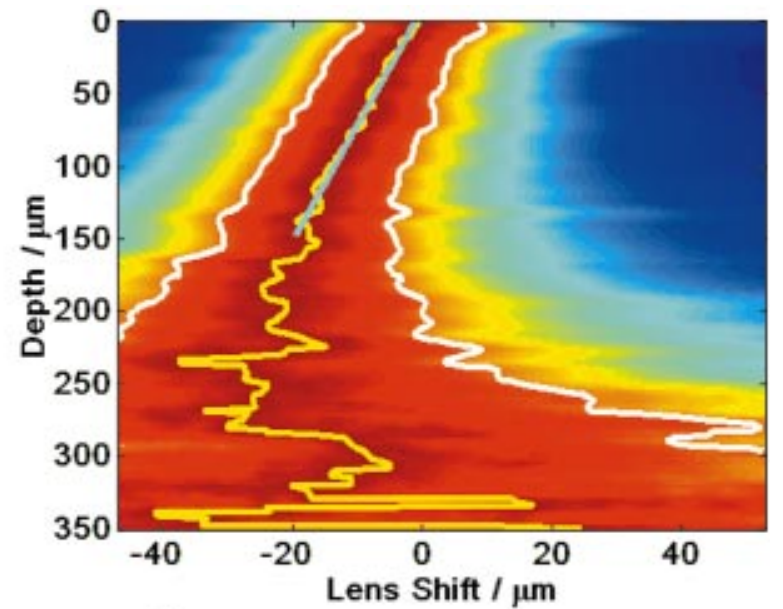

b)

Fig. 10 False color plots of the normalized ensemble intensity (human lower arm). 
of the MSA, which could be verified within the experimental error margin. Thus in a simplified picture, one can conclude that MSA is fairly independent of ambient skin conditions. On the other hand, given the results from the artificial polystyrene suspensions, one may be able to characterize different types of tissue or diseases via MSA where cell size distributions differ significantly.

The RI data have been shown to agree fairly well with the adjustment of the volume fractions of the suspensions. The higher the abundance of water, the lower the RI. This has also been observed in the pigskin experiment. In addition, the knowledge of RI provides an accurate scaling of the real (geometrical) depth within a sample.

It has already been demonstrated ${ }^{9}$ that the RI evaluation can be conducted on a layered or even local basis in turbid tissue. A basic problem is the appearance of speckles, which has to be taken into account. A viable approach is signal averaging over a certain volume of interest, which in turn sets resolution limits to such a RI evaluation. The same holds for the extraction of MSA data. Due to the higher complexity in the algorithm, however, the "decomposition" in segments is not a trivial undertaking and will require further scrutiny.

MSA and RI provide complementary information on the conditions of the turbid tissue. MSA exhibits some local scattering properties due to local variations in RI, which may lead to a (narrow) particle size distribution. RI measurements, on the other hand, provide an average value. It is conceivable that both parameters permit a more thorough understanding of tissue physiology under in vivo conditions.

There may be applications for MSA and/or RI measurements in cosmetics product evaluation and testing. It is also conceivable that both parameters could serve as additional "descriptors" for skin diagnoses in the clinical environment in the future. Certainly more studies need to be conducted.

\section{Acknowledgments}

The authors gratefully acknowledge the support from the ISIS colleagues in conducting the measurements with the OCT system SkinDex $300^{\circledR}$.

\section{References}

1. D. Huang, E. A. Swanson, C. P. Lin, J. S. Schuman, W. G. Stinson, W. Chang, M. R. Hee, T. Flotte, K. Gegory, C. A. Puliafito, and J. G. Fujimoto, "Optical coherence tomography," Science 254, 1178-1181 (1991).

2. A. F. Fercher, C. K. Hitzenberger, W. Drexler, G. Kamp, and H. Sattmann, "In vivo optical coherence tomography," Am. J. Ophthalmol. 116, 113-114 (1993).

3. A. Sergeev, V. Gelikonov, G. Gelikonov, F. Feldchtein, R. V. Kuranov, N. Gladkova, N. M. Shakhova, L. B. Snopova, A. V. Shakhov, I. A. Kuznetzova, A. N. Denisenko, V. V. Pochinko, Yu. P. Chumakov, and O. S. Streltzova, "In vivo endoscopic OCT imaging of precancer and cancer states of human mucosa," Opt. Express 1(13), 432-440 (1997).

4. J. M. Schmitt, M. Yadlowsky, and R. F. Bonner, "Subsurface imaging of living skin with optical coherence tomography," Dermatology (Basel, Switz.) 191, 93-98 (1995).

5. J. Welzel, E. Lankenau, R. Birngruber, and R. Engelhardt, "Optical coherence tomography of the human skin," J. Am. Acad. Dermatol. 37, 958-963 (1997).

6. J. M. Schmitt, S. H. Xiang, and K. M. Yung, "Differential absorption imaging with optical coherence tomography," J. Opt. Soc. Am. A 15, 2288-2296 (1998).

7. M. R. Hee, D. Huang, E. A. Swanson, and J. G. Fujimoto, "Polarization-sensitive low-coherence reflectometer for birefringence characterization and ranging," J. Opt. Soc. Am. A 9, 903-908 (1992).

8. Z. Chen, T. E. Milner, X. Wang, S. Srinivas, and J. S. Nelson, "Optical Doppler tomography: Imaging in vivo blood flow dynamics following pharmacological intervention and photodynamic therapy," Photochem. Photobiol. 67, 1-7 (1998).

9. A. Knuettel and M. Boehlau-Godau, "Spatially confined and temporally resolved refractive index and scattering evaluation in human skin performed with optical coherence tomography," J. Biomed. Opt. 5(1), 83-92 (2000).

10. C. F. Bohren and D. R. Huffman, Absorption and Scattering of Light by Small Particles, Wiley, New York (1983).

11. L. Thrane, H. Yura, and P. Andersen, "Analysis of optical coherence tomography systems based on the extended Huygens-Fresnel principle," J. Opt. Soc. Am. A 17(3), 484-490 (2000).

12. D. A. Boas, K. K. Bizheva, and A. M. Siegel, "Using dynamic lowcoherence interferometry to image Brownian motion within highly scattering media," Opt. Lett. 23(5), 319-321 (1998).

13. Klein/Furtak, Optik, Springer, Berlin (1988). 